\title{
MicroRNA in cardiovascular biology and disease
}

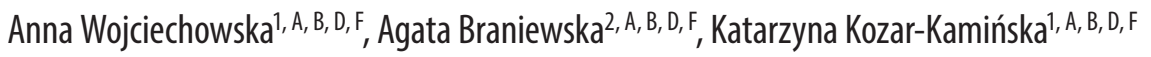 \\ ${ }^{1}$ Laboratory of Immunology, Department of Medical Biology, The Cardinal Stefan Wyszyński Institute of Cardiology, Warszawa, Poland \\ ${ }^{2}$ Department of Immunology, Medical University of Warsaw, Poland \\ A - research concept and design; $\mathrm{B}$ - collection and/or assembly of data; C - data analysis and interpretation; \\ $D$ - writing the article; $E$ - critical revision of the article; $F$ - final approval of article
}

\author{
Address for correspondence \\ Anna Wojciechowska \\ E-mail:awojciechowska@ikard.pl \\ Funding sources \\ None declared

\section{Conflict of interest} \\ None declared
}

Received on December 7, 2015

Revised on April 14, 2016

Accepted on April 27, 2016

\begin{abstract}
MicroRNAs (miRNAs) are members of a non-coding RNA family. They act as negative regulators of protein translation by affecting messenger RNA (mRNA) stability; they modulate numerous signaling pathways and cellular processes, and are involved in cell-to-cell communication. Thus, studies on miRNAs offer an opportunity to improve our understanding of complex biological mechanisms. In the cardiovascular system, miRNAs control functions of various cells, such as cardiomyocytes, endothelial cells, smooth muscle cells and fibroblasts. The pivotal role of miRNAs in the cardiovascular system provides a new perspective on the pathophysiology of disorders like myocardial infarction, hypertrophy, fibrosis, heart failure, arrhythmia, inflammation and atherosclerosis. MiRNAs are differentially expressed in diseased tissue and can be released into circulation. Manipulation of miRNA activity may influence the course of a disease. Therefore, miRNAs have become an active field of research for developing new diagnostic and therapeutic tools. This review discusses emerging functions of miRNAs in cardiogenesis, heart regeneration and the pathophysiology of cardiovascular diseases.
\end{abstract}

Key words: microRNA, cardiovascular disease, heart regeneration, heart development

DOI

10.17219/acem/62915

\section{Copyright}

Copyright by Author(s)

This is an article distributed under the terms of the

Creative Commons Attribution Non-Commercial License

(http://creativecommons.org/licenses/by-nc-nd/4.0/) 
MicroRNAs (miRNAs) are a class of single-stranded, non-coding RNAs, about 22 nucleotides in length, which negatively regulate gene expression at the post-transcriptional level. They bind to messenger RNA (mRNA) in a complementary way, and cause gene silencing through the inhibition of translation and/or degradation of mRNA. ${ }^{1}$ MiRNAs play a role in regulating various biological processes including embryogenesis, cell proliferation and differentiation, apoptosis or tumorigenesis. ${ }^{2}$ In the cardiovascular system, miRNAs control cardiomyocyte growth and contractility, the development and maintenance of cardiac rhythm, plaque formation, lipid metabolism and angiogenesis. ${ }^{2-5}$ Altered miRNA expression can be found in the blood of patients with various cardiovascular diseases, which makes them attractive candidates for noninvasive biomarkers. ${ }^{6,7}$ It has been estimated that miRNAs control the activity of $30-50 \%$ of protein-coding genes. ${ }^{7}$ Unlike transcriptional regulators, which have a turn-onand-off function in controlling gene expression, the varied profiles of miRNAs appear to fine-tune the level of protein expression to changes in environmental conditions. ${ }^{2}$ A single miRNA may influence the expression of hundreds of genes in a cell, and each mRNA molecule may be regulated by multiple miRNAs that interact or compete with each other. ${ }^{8}$ The first miRNA, lin-4, was discovered in the nematode Caenorhabditis elegans in 1993. ${ }^{1}$ To date, about 2500 miRNAs have been identified in the human genome. All known sequences of miRNAs are available in the database at the website www.mirbase.org.

\section{MicroRNA biogenesis and mechanisms of action}

MiRNA genes are an evolutionarily conserved integral part of the cell genome. They can be transcribed as independent transcription units in intergenic regions or in the introns and exons of protein-coding genes. MiRNA genes can exist individually or form polycistronic clusters containing multiple miRNA components. ${ }^{1}$ MiRNAs are transcribed in the nucleus by RNA polymerase II (RNA Pol II) to primary miRNAs (pri-miRNAs), which can be a few kilobases long. Pri-miRNAs are cleaved by a protein complex containing the RNase III endonuclease Drosha into approximately 70-nucleotide precursor miRNAs (pre-miRNAs) with a hairpin structure. ${ }^{1}$ Next, a GTP-dependent protein, exportin-5, recognizes a short stem of 2-3 nucleotides overhanging at the end of the pre-miRNAs and transports them from the nucleus to the cytoplasm. ${ }^{1}$ Alternatively, pre-miRNAs can be processed independently of the Drosha complex through the direct splicing of introns. ${ }^{6}$

In the cytoplasm, pre-miRNA is cleaved by the RNase III endonuclease Dicer to approximately 22-nucleotide double-stranded miRNAs. One strand, called a guide strand, is loaded onto the RNA-induced silencing complex (RISC) and becomes mature miRNA, while the other strand, called a passenger strand, is degraded or incorporated into microvesicles and released from the cell. ${ }^{9}$ Both mature and pre-miRNAs can be found in microvesicles. ${ }^{9}$ The formation of the RISC effector, which contains Argonaute 2 (Ago2) protein, allows miRNAs to bind to target mRNAs. ${ }^{1,10}$

The RISC binding sites are complementary sequences present mainly in the 3'-untranslated region (3'-UTR) of mRNAs. In cases of perfect complementarity of the miRNA-mRNA sequences, Ago2 protein, which has endonuclease activity, cleaves the mRNA, leading to its degradation. Mismatches in the sequence inhibit translation (Fig. 1). ${ }^{1}$

Most miRNAs are localized intracellularly, but some of them are released into the blood in association with proteins (e.g. Ago2, nucleophosmin 1 and HDL) or as a component of cell-derived microvesicles (e.g. exosomes or apoptotic bodies). MiRNAs may be released in response to cell activation stimuli, injury or after cell death. ${ }^{9}$ In the circulation, miRNAs are transported to distant sites and interact with cells by fusing with the cell membrane or through receptor-mediated binding, which suggests that miRNAs play a role in cell-to-cell communication., ${ }^{9,10}$ For example, in response to tissue damage, miR-126 is transported in endothelial cell-derived apoptotic bodies to vascular smooth muscle cells (VSMCs), where it mediates the synthesis of the CXCL12 chemokine to recruit progenitor cells and provide vascular protection. ${ }^{5}$

\section{The nomenclature of microRNAs}

With the exception of a few miRNAs that were discovered early (such as the let family), the nomenclature of mature miRNAs consists of the prefix "miR" and the identifying number, e.g. miR-499. Pre-miRNAs are indicated by italics and the lower case prefix "mir". Three- or four-letter prefixes indicate the species, e.g. hsa-miR-101 in Homo sapiens. An additional lower case letter is appended to miRNAs with similar sequences, differing by only one or two nucleotides, e.g. miR-123a or miR-123b. ${ }^{6}$ If two pre-miRNAs that are located at different sites in the genome lead to an identical mature miRNA, the miRNA is annotated with an additional hyphen and number, e.g. miR-194-1 or miR-194-2. Two different miRNAs that originate from the same precursor are named according to their location on the hairpin: miR-17-5p (5' arm) or miR-17-3p (3' arm), or based on their level of expression: miR-123 or miR-123\%. An asterisk indicates the miRNA strand that is expressed at a lower level. ${ }^{6}$

\section{Controlling microRNA activity}

The activity of miRNAs can be modulated by two different approaches based on mimicking miRNA functions 
Fig. 1. A schematic representation of the biogenesis of miRNAs and their mechanism of action: a miRNA gene is transcribed by RNA polymerase II (RNA Pol II) to stem-loop primary miRNAs (pri-miRNAs). Within the nucleus, pri-miRNA is processed by the RNase III endonuclease Drosha into hairpin-like precursor miRNAs (pre-miRNAs). Alternatively, pre-miRNAs are processed independently of the Drosha complex, through direct splicing of introns, to form pre-miRNAs called mirtrons. The pre-miRNAs/mirtrons are then transported to the cytoplasm by a GTP-dependent protein transporter, exportin-5. Within the cytoplasm, pre-miRNAs are cleaved by the RNase III endonuclease Dicer to approximately 22-nucleotide double-stranded miRNAs. After unwinding, both miRNA strands can be functional; however, usually one of them, termed the guide strand, is incorporated into the RNA-induced silencing complex (RISC). MiRNA-RISC complexes containing Argonaute 2 (Ago2) protein bind to the 3'-untranslated region (3'-UTR) of target mRNAs and causes gene silencing by inhibiting translation and/or through mRNA degradation

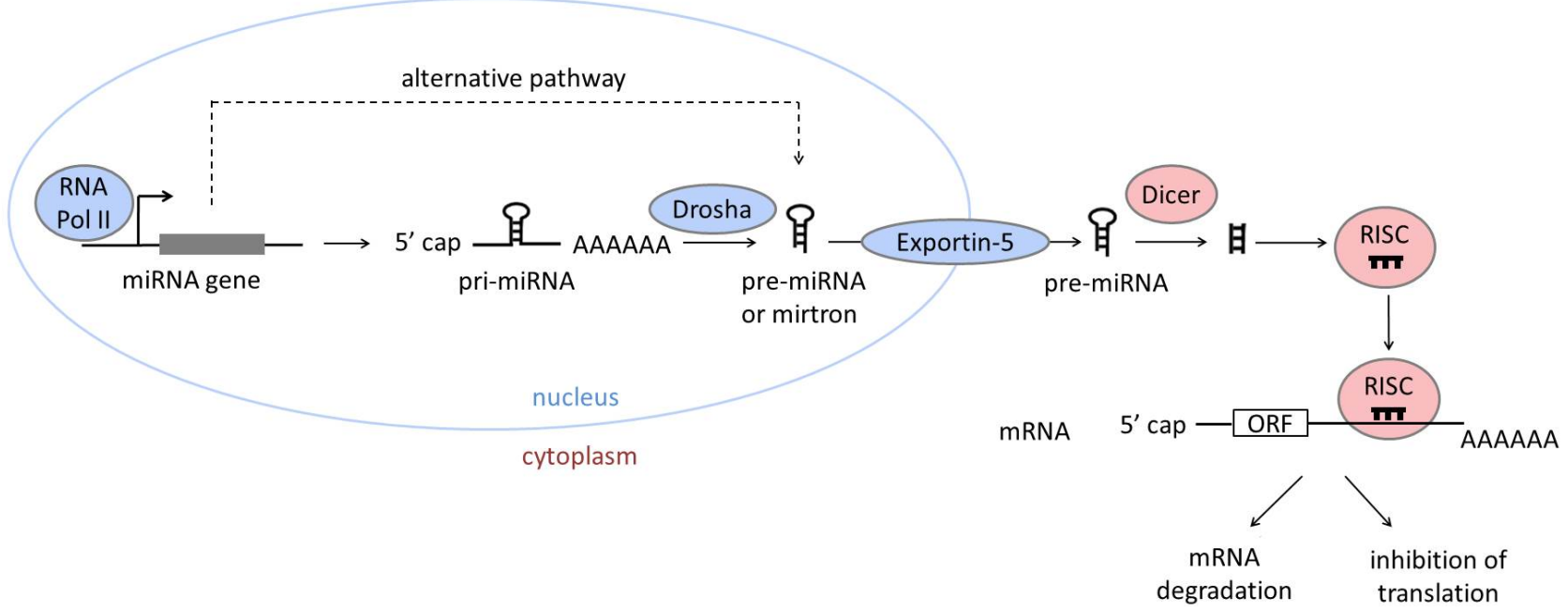

Fig. 2. Different approaches to targeting miRNA activity. (1) Endogenous miRNA (blue) binds to a complementary sequence known as a seed sequence (blue box) present in the $3^{\prime}$-untranslated region (3'-UTR) of mRNA. (2) A miRNA mimic (red) is a chemically synthesized double-stranded RNA molecule. This structure imitates endogenous miRNA and targets complementary mRNA. (3) An antagomiR (black) is a synthetic oligonucleotide that is complementary to a particular miRNA. This oligonucleotide binds to miRNA and inhibits its action. (4) MiRNA sponge (green) contains several seed sequences for a particular miRNA. Delivery to the cell results in binding with the target miRNAs and reduces the number of free and active miRNAs

\section{1. endogenous miRNA}
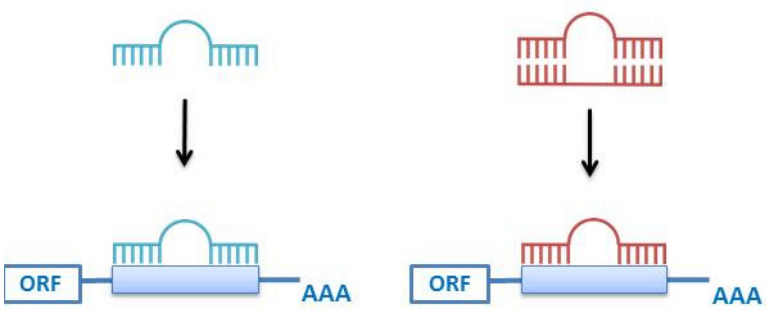

2. miRNA mimic

AAA

or silencing its action (Fig. 2). ${ }^{3}$ In cases of compromised miRNA levels, exogenous miRNAs can be administrated in vivo. MiRNA mimics are small, chemically synthesized double-stranded RNAs that imitate endogenous miRNAs and cause gene silencing. One strand of this molecule is identical to the native form of miRNA, the other is complementary. The double-stranded structure is required so that the RISC can recognize miRNA mimics accurately. ${ }^{11}$

AntagomiRs are synthetic RNA molecules used to silence aberrantly expressed miRNAs. AntagomiRs function by binding to miRNAs and inhibiting their actions. They are complementary to the sequence of a full-length
3. antagomiR

4. miRNA sponge

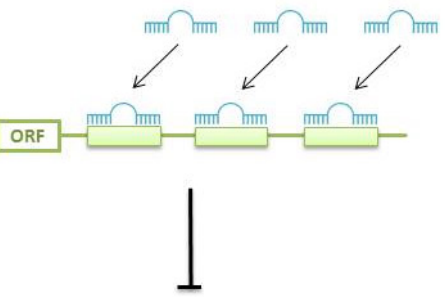

ORF
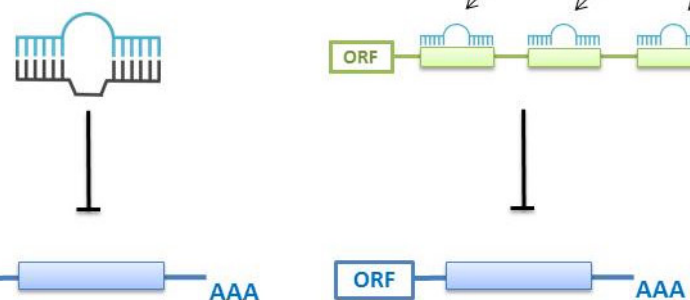

sequence or a seed sequence (a 28-nucleotide-long mRNA binding site) of a specific miRNA. They are chemically modified to improve cellular uptake, in vivo stability and affinity to miRNA. ${ }^{11}$ MiRNA sponges are another approach to reducing miRNA levels. MiRNA sponges are transcripts that contain multiple complementary regions for miRNAs that have the same target site. Delivering miRNA sponges to a cell results in their binding with the target miRNAs and reduces the number of free and active miRNAs. $^{11}$

The highly conserved miRNA sequence facilitates the adaptation of results from studies in animal models to 
clinical settings. However, the use of miRNA-targeting drugs still remains challenging. To enter the cell, oligonucleotides must pass the lipid bilayer of the cell membrane. They are therefore chemically modified to improve cellular uptake and in vivo stability. Changes include modifications in 2' sugar (e. g. 2'-OMe, 2'-MOE, 2'-F) or conjugation with cholesterol. Bicyclic nucleotides called locked nucleic acid (LNA) have enhanced affinity to miRNAs and stability. ${ }^{11}$ Another method is delivering antisense nucleotides by means of supramolecular nanoparticles, such as liposomes or polymeric nanoparticles. ${ }^{12}$ Unlike conventional drugs, which are specific for one cellular target (e.g. an enzyme or a receptor), one miRNA can modulate a number of target genes in different cells in a pathway, which can provide greater therapeutic effects. However, this feature could also be a disadvantage, because it can lead to undesired effects. Understanding this effect is an important step in developing miRNA-based therapies. $^{12}$

\section{MicroRNAs in heart development}

The importance of miRNAs in heart biology was revealed by blocking the expression of all the miRNAs in the cardiovascular system. ${ }^{2}$ This effect was obtained through tissue-specific deletion of genes essential to miRNA biogenesis, such as Drosha, DGCR8 (coding for a protein which forms a complex with Drosha), Ago2 or Dicer. Deletion of these critical genes in mice resulted in death during early gestation due to severe developmental defects of the heart and blood vessels. However, deleting genes for individual miRNAs is not lethal.

Different types of cells are characterized by their specific profile of miRNA expression. Interestingly, only 18 miRNA families account for approximately $90 \%$ of cardiac miRNAs. ${ }^{13}$ Among cardiomyocytes, miR-1 is the most abundant one. MiR-1 and miR-133 arise from the same bicistronic transcript, but miR-133 expression is lower. MiR-1 and miR-133 cooperatively promote mesoderm differentiation in embryonic stem cells (ESCs). Later in their development, they play opposite roles: miR-1 promotes and miR-133 inhibits differentiation of mesoderm into cardiomyocytes. ${ }^{14}$ MiR-1 influences cardiogenesis by regulating the expression of transcription factors Irx 5 and Hand2. ${ }^{15}$ The Hand 2 protein is involved in the development of the outflow tract and right ventricle. ${ }^{16}$ Targeted deletion of miR-1 results in ventricular septal defects (VSDs). ${ }^{15}$ The Irx 5 protein regulates the expression of potassium channel genes like potassium voltagegated channel subfamily D member 2 (Kcnd2) and determines the cardiac ventricular repolarization gradient. ${ }^{15}$ MiR-133 influences the activity of serum response factor (SRF) and cyclin D2, the transcription factors involved in cell cycle progression. SRF regulates the genes responsible for cardiac muscle and smooth muscle differentiation and growth; it also activates transcription of miR-133/1. Cyclin D2 controls cardiomyocyte proliferation by acting on the phosphorylation of retinoblastoma protein in the G1 phase of the cell cycle. ${ }^{17}$

Contractile protein expression is strictly regulated during heart development. Abnormal synthesis of myosin genes underlies pathological cardiac remodeling. Myosin genes remain under the control of miR-208a, miR208b and miR-499, which are encoded in the introns of Myh6 (alpha-myosin heavy chain, $\alpha-\mathrm{MHC}$ ), Myh7 (beta-myosin heavy chain, $\beta-\mathrm{MHC}$ ) and $M y h 7 b$, respectively. ${ }^{14}$ In rodents, $\beta$-MHC (a slow ATPase) expression occurs during embryonic development, $\alpha-\mathrm{MHC}$ (a fast ATP-ase) after birth, while $M y h 7 b$ expression occurs at both stages. ${ }^{18}$ The fact that miR-208a and miR-208b have identical seed sequences suggests that they regulate common target genes at different stages of development. In the adult heart, $\beta$-MHC synthesis is re-expressed in cardiomyocytes under stress conditions such as hypoxia or hypothyroidism. ${ }^{14}$ Deletion of miR-208a results in ectopic expression of fast skeletal muscle genes and impaired postnatal stress response. ${ }^{19}$

The miR-15 family consists of $\mathrm{miR}-15 \mathrm{a} / \mathrm{b}$, miR-161/2, miR-195 and miR-497, which have identical seed sequences. MiR-195 is upregulated immediately after birth and halts cardiomyocyte proliferation; it controls numerous cell cycle genes, including checkpoint kinase 1 (Chek1). Overexpression of miR-195 results in VSDs and ventricular hypoplasia. ${ }^{20} \mathrm{MiR}-15 \mathrm{~b}$ controls ATP level in cardiomyocytes by targeting Arl2, a component of the ADP/ATP exchanger in mitochondria. ${ }^{21}$

The miR-17 92 cluster consists of miR-17, miR-18a, miR-19a/b, miR-20a and miR-92. MiR-17 92 transcription is activated by the bone morphogenetic protein (BMP) signaling pathway. Through downregulation of Isl1 and TbxI, miR-17 92 promotes the development of the cardiac outflow tract and the differentiation of the second heart field (SHF) progenitors into right ventricle myocytes. ${ }^{22}$ Deletion of miR17 92 leads to VSDs and lung hypoplasia, and consequently to death. These effects are attributed in part to the upregulation of pro-apoptotic proteins like Bim, which is a target gene of this miRNA cluster. ${ }^{22}$

The role of miRNAs during various stages of heart development is schematically represented in Fig. 3.

\section{MicroRNAs in heart regeneration}

Neonatal murine hearts have been demonstrated to regenerate following infarction. Ligation of the left anterior descending (LAD) artery in a one-day-old mouse results in necrosis of about $75 \%$ of the heart muscle. Within several weeks, spontaneous regeneration of the anterior wall leads to the recovery of the systolic function. Overexpression of miR-195 has been shown to impair myocardial regeneration and cause massive cardiac fibrosis. ${ }^{23}$ 
Fig. 3. A schematic representation of mouse cardiac morphogenesis and the role of miRNAs in this process. The colors represent the contribution of different precursor pools to the forming chambers. The heart is of mesodermal origin. At embryonic day 7.5 (E7.5), 2 populations of cells, termed the first heart field (FHS) and the second heart field (SHF), build up the cardiac crescent. Subsequently, the cells migrate and the linear heart tube is formed at E8.0. Shortly thereafter, the heart tube starts spontaneous contractions and supports the blood supply of the developing embryo. Finally, the process of cardiac looping and a series of morphological changes contribute to the formation of the four-chambered heart by E10.5. The progressive septation of the atria, ventricles and the common outflow track then takes place

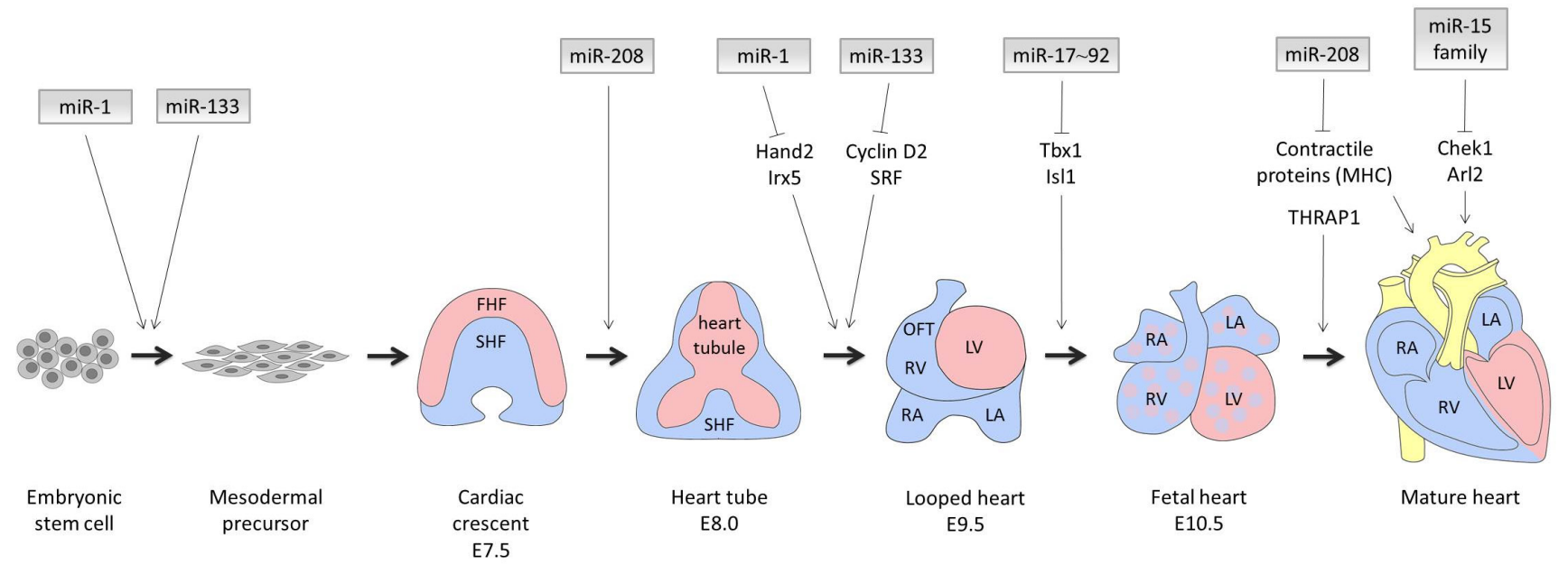

Inhibition of the miR-15 family promotes myocyte proliferation and ameliorates cardiac functions in adults after myocardial infarction. ${ }^{23} \mathrm{MiR}-133$ is another molecule regulating the cell cycle of cardiomyocytes. Following resection of $20 \%$ of the zebrafish ventricular apex, proliferation of the remaining cardiomyocytes occurs, which leads to complete heart regeneration. Studies have noted reduced expression of miR-133 in regenerating zebrafish heart. Upregulation of miR-133 diminishes its regenerative potential, whereas decreasing the level of miR-133 with specific miRNA sponges promotes regeneration. ${ }^{24}$ To sum up, the miR-15 and miR-133 families attenuate the regenerative capacity of the heart by inhibiting cardiomyocyte proliferation.

MiR-199 and miR-590 have been found to induce cell cycle re-entry in cardiomyocytes. Intracardiac administration of these molecules into the infarct border zone stimulates cardiomyocyte proliferation in adult mice. MiR-199 and miR-590 have been shown to promote regeneration of the myocardium and to improve cardiac function. ${ }^{25}$

Stem cell-based therapies represent an attractive approach for treating cardiovascular diseases. However, regenerative therapy based on delivering stem cells into the heart has not yet been successful in clinical trials. Nowadays, many studies aim to improve the effects of this therapy by increasing the survival of cells transplanted into the infarcted region and enhancing the differentiation of stem cells into cardiomyocytes. ${ }^{26} \mathrm{MiR}-1$ and miR133 have been found to induce the differentiation of stem cells and heart progenitor cells into cardiomyocytes. ${ }^{14}$ Moreover, transplanting stem cells overexpressing miR-1 into the infarcted zone increases cardiomyocyte differentiation, promotes regeneration and improves cardiac function. ${ }^{27}$ Similar results are achieved by transplanting c-kit+ cardiac progenitor cells overexpressing miR-499. ${ }^{28}$

Cardiac fibrosis following myocardial infarction, resulting from excessive fibroblast activation, reduces regeneration, leads to pathological remodeling, impairs systolic function and increases susceptibility to arrhythmias. It has been shown that the expression of a suitable combination of transcription factor genes (Gata4, Mef2c, Tbx5) could convert resident cardiac non-myocyte fibroblasts into contractile cells by direct reprogramming. ${ }^{29}$ A similar effect can be obtained when miRNAs are used. Lentiviral-mediated delivery of miR-1, miR-133, miR-208 and miR-499 into the infarct border zone has been found to induce direct fibroblast reprogramming into cardiomyocytes in situ. ${ }^{30}$ Reprogrammed cardiomyocytes express cardiac markers and sarcomeric organization, and have electrophysiological properties characteristic of mature ventricular cardiac myocytes. Moreover, reprogramming is associated with an improvement in fractional shortening, suggesting the functional recovery of the damaged myocardium. ${ }^{31}$

Fig. 4 summarizes the role of miRNAs in cardiac regeneration: regulation of cardiomyocyte proliferation, stem or progenitor cell differentiation and direct reprogramming of fibroblasts.

\section{MicroRNAs in heart diseases}

\section{Myocardial infarction and cardiac remodeling}

Ischemia/reperfusion injury associated with myocardial infraction leads to remodeling in the myocardium, which is regulated by various miRNAs. Activation of 
Fig.4. MiRNA activity in the process of myocardial regeneration. Three approaches to cardiac muscle regeneration are presented: regulation of cardiomyocyte proliferation; stem or progenitor cell differentiation; and direct reprogramming of fibroblasts

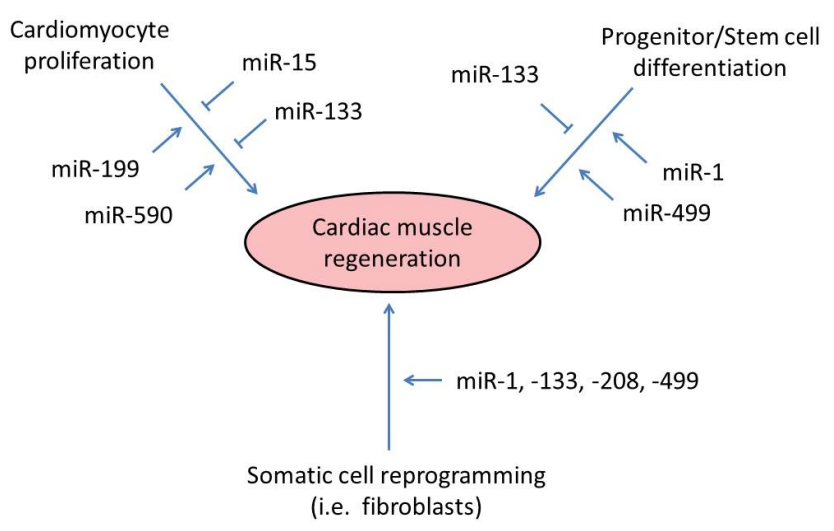

stress signaling pathways triggers changes in miRNA expression; $\mathrm{miR}-24, \mathrm{miR}-320$ and $\mathrm{miR}-29$ are downregulated in myocardial infarction. MiR-24 inhibits translation of Bim, a proapoptotic protein. Restoration of miR24 to physiological levels by specific miRNA mimics attenuates apoptosis and decreases scar size. ${ }^{2}$ Proapoptotic properties are attributed to miR-320, which negatively regulates heat shock protein 20 (HSP20), which functions as a cell protector after an ischemic injury. ${ }^{2}$ MiR-29 controls genes encoding collagen (COL1A1, COL1A2, COL3A1) and extracellular matrix proteins, including fibrillin (FBN1) and elastin (ELN1). Low expression of miR-29 after myocardial infarction results in scar formation..$^{32}$ In addition, miR-199 is downregulated in cardiac myocytes during oxygen deprivation. This induces its target genes: hypoxia-inducible factor- $1 \alpha$ $(H I F-1 \alpha)$ and sirtuin 1 (Sirt 1), and subsequent activation of hypoxia-triggered pathways. Restoration of physiological miR-199 levels inhibits HIF-1 $\alpha$ expression and its stabilization of p53, a tumor supressor responsible for sustaining the genome integrity, which leads to a reduction in apoptosis. ${ }^{33}$

Expression of the miR-15/16 family and miR-499 increases after myocardial infarction. ${ }^{4,34}$ The miR-15/16 family regulates cardiomyocyte proliferation and survival in response to injury, and its inhibition protects cardiomyocytes from apoptosis. ${ }^{34}$ MiR-499 influences cardiomyocyte apoptosis by downregulating calcineurin and dynamin-related protein 1 (Drp1), which are involved in mitochondrial fission. According to the literature, the upregulation of miR-499 reduces apoptosis and infarct size, while miR-499 knockdown has the opposite effect. ${ }^{4}$ In contrast, another report showed that miR-499 overexpression in the heart can lead to cardiomyocyte hypertrophy and cardiomyopathy, suggesting discrepancies that may be caused by acute or chronic modulation of miRNA. ${ }^{4}$ MiR-214, which increases in mouse and human tissue after myocardial infarction, exerts a protective effect during ischemia/reperfusion. It reduces calcium overload and promotes cardiomyocyte survival through inhibition of the sodium/calcium exchanger (NCX1) and Bim. Deletion of miR-214 increases injury and mortality following myocardial infarction. ${ }^{4}$

At the molecular level, cardiac remodeling is accompanied by a gene expression switch from the adult $\alpha-M H C$ isoform to the fetal $\beta-M H C$. MiR-208a, which is encoded in the intron of the $\alpha-M H C$ gene, has been shown to be involved in this process, inducing cardiomyocyte hypertrophy, fibrosis and increasing $\beta-M H C$ expression. Deletion of miR-208a protects the heart from pathological remodeling under stress conditions. Thyroid-hormonereceptor-associated protein 1 (THRAP1) is considered a target gene for miR-208a. ${ }^{3,34}$

MiR-21 promotes myocyte hypertrophy and fibrosis by repressing the Sprouty2 transcription factor, which controls the pro-fibrotic extracellular signal-regulated kinase-mitogen-activated protein kinase (ERK-MAPK) pathway. Specific antagomiR-mediated inhibition of miR21 blocks this cascade and results in a reduction in both hypertrophy and fibrosis. ${ }^{13}$ However, genetic deletion of miR-21 does not alter the pathological cardiac response to pressure overload. ${ }^{34}$ This discrepancy indicates that miR-21 plays a complex role in the pathophysiology of heart diseases, which requires further investigation. Another possibility may be the existence of a compensatory mechanism revealed under a permanent miR-21 knockdown. ${ }^{34}$

\section{Heart failure}

Impaired cardiac contractile function caused by disrupted calcium handling is a hallmark of heart failure. Recently, Cai et al. demonstrated that miR-765 is overexpressed in failing hearts and is involved in contractile regulation. MiR-765 contributes to increased protein phosphatase 1 (PP-1) activity and the subsequent dephosphorylation of key calcium cycling proteins by silencing its endogenous inhibitor- $1{ }^{35}$ Likewise, miR-25 is upregulated in failing hearts and controls myocyte contractile function by repressing the sarcoplasmic reticulum calcium uptake pump, SERCA2a. Anti-miR-25 delivery restores cardiac function and improves survival. ${ }^{36}$ Also, miR-24 regulates calcium homeostasis through Junctophilin-2 repression, which results in decreased efficiency of excitation-contraction (E-C) coupling in cardiomyocytes. ${ }^{37}$

In a recent paper, Melman et al. provided evidence that increased cardiac miR-30d expression has a significant impact on responses to cardiac resynchronization therapy (CRT), and that plasma levels of miR-30d may correlate with responsiveness to CRT in heart failure patients. MiR-30d is regulated by mechanical stretch, and is released in exosomes by cardiomyocytes. It protects cardiomyocytes from TNF- $\alpha$-elicited inflammation and cell 
death by targeting the MAP4K4 protein or, possibly via other indirect pathways, resulting in beneficial cardiac remodeling. ${ }^{38}$

Potous et al. revealed the role of miR-126 in right ventricle (RV) failure associated with pulmonary arterial hypertension (PAH). Due to the methylation process, miR-126 is downregulated in RV failure in PAH patients, causing decreased capillary density. MiR-126 is expressed in endothelial cells and directly targets Sprouty-related EVH1 domain-containing protein 1 (SPRED-1), a negative regulator of the vascular endothelial growth factor (VEGF) signaling pathway. Administration of miR-126 mimics ameliorates microvascular density, improves RV function and diminishes fibrosis, whereas antagomiR-mediated miR-126 downregulation exacerbates RV failure. ${ }^{39}$

Recent research by Halkein et al. proposed the $16-\mathrm{kDa}$ $\mathrm{N}$-terminal fragment of the nursing hormone prolactin (16K PRL) as a potential factor initiating and driving peripartum cardiomyopathy (PPCM). Expression of miR-146a is induced in endothelial cells (ECs) by 16K PRL. By targeting the NRAS gene, miR-146a reduces the proliferation and viability of ECs and leads to the destruction of the cardiac microvasculature. Moreover, 16K PRL promotes the transfer of miR-146a-loaded exosomes from ECs to cardiomyocytes, which results in diminished overall metabolic activity and increased vulnerability to apoptosis. In cardiomyocytes, miR-146a also downregulates Erbb4, Notch1 and Irak1. Post-natal knockout of ErbB2 in mice leads to dilated cardiomyopathy, which indicates that ErbB signaling plays an essential role in the physiological status of the adult heart. Pharmacological inhibition of miR-146a or 16K PRL attenuates ErbB4 downregulation and improves cardiac function. Increased miR-146a and decreased ErbB4 expression has been reported in the heart muscle of PPCM patients, suggesting that increased miR-146a and decreased ErbB4 expression contributes to the development of PPCM in humans. ${ }^{40}$

\section{Arrhythmias}

MiR-1 and miR-133 play an important role in the pathophysiology of arrhythmias. MiR-1 is upregulated in ischemic myocardium, and contributes to the slowdown of cardiac conduction by and depolarization of the cytoplasmic membrane. The arrhythmogenic properties of miR1 include repression of GJA1 and KCNJ2, which encode the connexin43 and Kir2.1 subunits of the $I_{\mathrm{K} 1}$ channel, respectively. ${ }^{41}$ Moreover, overexpression of $\mathrm{HCN} 2$ and HCN4, which are regulated by miR-1 and miR-133, support arrhythmia-prone mechanisms. HCN2 and HCN4, which belong to the hyperpolarization-activated cyclic nucleotide-gated channel $(H C N)$ gene family, are found in pacemaker, atrial and ventricular cells. Age-associated low levels of miR-1 and miR-133 contribute to the overexpression of HCN2 and HCN4, and this results in abnormal cardiac electrical activity. ${ }^{42}$

\section{Hypertension}

MiRNAs target genes in the renin-angiotensin-aldosterone system (RAAS), which is crucial in blood pressure regulation. MiR-155 has been found to regulate expression of the angiotensin II type 1 receptor, AGTR1. AGTR1 correlates negatively with miR-155 and positively with blood pressure. Inhibition of miR-155 in Chinese hamster ovary cells resulted in upregulation of AGTR1 and ERK1/2 activation. ${ }^{43}$ Furthermore, the miR-155 gene is located on chromosome 21, and trisomy 21 is associated with decreased blood pressure. An analysis of monozygotic twins revealed low AGTR1 protein expression and upregulation of miR-155 in patients with trisomy 21. An AGTR1 allele with a single nucleotide polymorphism (SNP) in the 3'-UTR region $(+1166 \mathrm{~A} / \mathrm{C})$ is not recognized by miR-155. This SNP is associated with an increased risk of essential hypertension..$^{44}$ Moreover, blood pressure alterations have been linked with SNPs located on the miRNA-binding site of other RAAS genes: the arginine vasopressin 1A receptor (AVPR1A), bradykinin receptor 2 $(B D K R B 2)$ and thromboxane $\mathrm{A} 2$ receptor $(T B X A 2 R))^{4,44}$

Pressure overload induces overexpression of miR-23a through activation of the nuclear factor of activated $\mathrm{T}$ cells (NFAT) transcription factor NFATc3. This results in inhibition of the anti-hypertrophic molecule-muscle specific ring finger protein 1 (MuRF1). MuRF1 in turn halts NFATc3, establishing a positive feedback loop. Downregulation of miR-23a with a specific antagomiR prevents cardiac hypertrophy. NFATc3 has also been shown to induce miR-199 expression. MiR-199 targets the kinase Dyrk1a, which inhibits NFATc3. It is noteworthy that miR-199 inhibition does not only prevent pathological hypertrophy, but also reverses cardiac remodeling. ${ }^{34}$

\section{MicroRNAs in vascular homeostasis and diseases}

As components of vessel walls, ECs and smooth muscle cells (SMCs) take part in maintaining vascular homeostasis. MiR-10a controls a pro-inflammatory EC phenotype by regulating the expression of adhesion molecules, and miR-10a expression is decreased in atherosusceptible regions of the aorta. ${ }^{3}$ MiR-10a knockdown enhances the synthesis of such pro-inflammatory mediators as monocyte chemotactic protein-1 (MCP-1), interleukin-6 (IL-6), IL-8, vascular cell adhesion molecule-1 (VCAM-1) and E-selectin. Other molecules involved in regulating inflammation include miR-17-3p, miR-31, miR-126 and miR-181. ${ }^{3}$ Recent evidence suggests that miRNA Let-7g has a pleiotropic effect on ECs. Let-7g targets genes in the transforming growth factor $(T G F-\beta)$ signaling pathway and Sirt 1, a protein involved in cell senescence. Let-7g has been demonstrated a key anti-inflammatory and anti-aging molecule. Downregulation of Let-7g leads to en- 
dothelial activation and subsequent vessel injury. Moreover, low serum levels of Let-7g have been associated with increased circulating plasminogen activator inhibitor-1 (PAI-1). ${ }^{45}$

Vascular injury triggers phenotypic changes (de-differentiation) in VSMCs. An altered phenotype is characterized by improper contractility, increased proliferation and migration, which result in restenosis. ${ }^{13}$ Upon arterial injury, miR-143 and miR-145 are downregulated, whereas miR-21 is upregulated. Restoration of physiological miRNA levels protects VSMCs from de-differentiation and restenosis. ${ }^{13}$ MiR-145 controls neointimal lesion formation by silencing Kruppel-like factor 5 (KLF5) and its downstream molecule, myocardin. ${ }^{3}$. MiR-21 function is mediated by a tumor suppressor known to negatively regulate Akt/PKB signaling pathway PTEN and anti-apoptotic protein $\mathrm{Bcl}-2$. MiR-221 promotes VSMC proliferation by repressing the cyclin-dependent kinase inhibitor p27 Kip1 and reduces expression of contractile genes $\alpha$-smooth muscle actin (SMA), smooth muscle calponin (CNN),

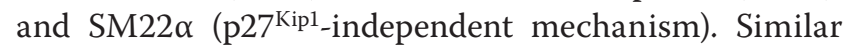
effects are caused by miR-26, which downregulates extracellular signal transducers Smad1 and Smad4 in the bone morphogenetic protein (BMP) signaling pathway. ${ }^{2}$

Zhao et al. reported the miR-143/145 expression in SMCs is induced by ECs. MiR-145 targets TGF- $\beta$ receptor II (TGFBR2) and regulates TGF- $\beta$ signaling in a selective manner: MiR-145 diminishes expression of matrix genes, while smooth muscle differentiation genes remain unaffected. ${ }^{46}$ On the other hand, when stimulated by cellto-cell contact, EC-derived TGF- $\beta$ mediates miR-143 and miR-145 transfer from SMCs to ECs through membrane protrusions known as tunneling nanotubes. This decreases the ability of ECs to form capillary-like structures and lowers their proliferation index, which leads to vessel stabilization. MiR-143 and miR-145 target hexokinase II (HKII) and integrin $\beta 8$ (ITG $\beta 8)$ genes, respectively. ${ }^{47}$

MiR-145 has been also implicated in the pathophysiology of atherosclerosis due to its effect on VSMC proliferation and phenotypic changes. In endothelial cells miR-143/145 expression is mediated by shear-responsive transcription factor - Kruppel like factor 2 (KLF2). ECs release extracellular vesicles (EVs) containing miR$143 / 145$, which are absorbed by SMCs to control target genes and act as atheroprotective molecules. Administering EC-derived EVs enriched with miR-143/145 results in a reduction in atherosclerotic lesion formation in the aortas of apolipoprotein E-deficient mice. ${ }^{4}$ An increase in miR-145 expression reduces plaque size in the aortic sinuses, diminishes the necrotic core and promotes collagen synthesis; these phenomena lead to plaque stabilization. ${ }^{3}$ On the other hand, neoangiogenesis and atherosclerotic plaque hemorrhage increase the subject's susceptibility to plaque rupture and clot formation, and miR-222/221, the miR-155 family and the miR-17 92 family are involved in the process. ${ }^{3}$ Macro- phages are the main effector cells in atherogenesis, as they promote inflammatory response, degrade lipoproteins and phagocyte cell debris. Cholesterol-loaded macrophages produce VEGF, a proangiogenic cytokine, and the miR-155 family, the miR-17 92 family and miR-222/221 regulate this process. Moreover, miR-342$-5 \mathrm{p}$ activates macrophages by inhibiting Akt1 kinase. ${ }^{3}$

Considering the important role of cholesterol in the pathophysiology of atherosclerosis, it is worth mentioning miR-122 and miR-33, which have been described as regulators of lipid homeostasis. ${ }^{3}$ MiR-122 is highly expressed in the liver, where it is involved in fatty acid oxidation and lipid synthesis. Downregulation of miR-122 results in decreased levels of both HDL and LDL cholesterol. ${ }^{4}$ MiR-33a and miR-33b are encoded in the introns of the sterol regulatory element-binding protein genes SREBP1 and SREBP1, respectively. MiR-33a targets ATPbinding cassette transporter $\mathrm{A} 1(A B C A 1)$ and inhibits cellular cholesterol export. Because 3'UTR mouse and human $A B C A 1$ genes possess several miR-33a binding sites, mRNA repression is strong. In addition, miR-33a/ miR-33b regulate $N P C 1$ and $A B C G 1$, which are also involved in cholesterol trafficking ${ }^{48}$; as well as $C R O T, C P$ $T 1 a, H A D H B$ and $A M P K a$, which are engaged in fatty acid oxidation. ${ }^{4}$ Inhibition of miR-33a/b upregulates ABCA1 expression in hepatocytes and macrophages, and leads to increased total cholesterol and HDL levels in serum. ${ }^{4}$ Interestingly, both strands of the miR-33 locus act together in lipid metabolism regulation, since miR-33a* and $\mathrm{miR}-33 \mathrm{~b} *$ repress genes similar to those targeted by $\mathrm{miR}-33 \mathrm{a} / \mathrm{b} .{ }^{48}$

\section{Conclusions}

The discovery of miRNA has changed our understanding of the regulation of gene expression. In the cardiovascular system, miRNAs control the proliferation and differentiation of stem and progenitor cells, and the function of cardiac myocytes, pacemaker cells, endothelial cells and smooth muscle cells. MiRNAs play a crucial role in cardiac development and regeneration. They are involved in cardiovascular pathophysiology and their expression is altered in various cardiovascular diseases. Modulation of miRNA expression may indeed change the course of a disease. The encouraging results of miRNA applications in experimental settings and reports of negligible toxicity to healthy tissues suggest that these molecules have considerable therapeutic potential. ${ }^{3}$

Currently, only two chemically modified oligonucleotides have been used in clinical trials. An antagomiR directed against miR-122 has completed the second phase of clinical trials. This oligonucleotide is used to treat hepatitis $\mathrm{C}$ virus (HCV). MiR-122 is specific to liver cells and is required for $\mathrm{HCV}$ replication. Delivering the antisense inhibitor of miR-122 reduces the number of viral copies 
without evidence of treatment resistance. ${ }^{49}$ Another molecule, MRX34, which mimics miR-34, has recently (at the time of writing) entered phase I clinical trials for the treatment of primary liver cancer. MiR-34 inhibits multiple oncogenic pathways and induces apoptosis in tumor cells. ${ }^{50}$

MiRNA studies represent an attractive and promising field of investigation. Identifying and understanding the role of miRNAs is an important step in the development of new therapeutic and diagnostic tools.

\section{References}

1. Wahid F, Shehzad A, Khan T, Kim YY. MicroRNAs: Synthesis, mechanism, function, and recent clinical trials. Biochim Biophys Acta. 2010;1803:1231-1243.

2. Hata A. Functions of microRNAs in cardiovascular biology and disease. Annu Rev Physiol. 2013;75:69-93.

3. Condorelli G, Latronico MV, Cavarretta E. MicroRNAs in cardiovascular diseases: Current knowledge and the road ahead. J Am Coll Cardiol. 2014;63:2177-2187.

4. Quiat D, Olson EN. MicroRNAs in cardiovascular disease: From pathogenesis to prevention and treatment. J Clin Invest. 2013;123:11-18.

5. Zernecke A, Bidzhekov K, Noels H, et al. Delivery of microRNA-126 by apoptotic bodies induces CXCL12-dependent vascular protection. Sci Signal. 2009;2, ra81.

6. Bronze-da-Rocha E. MicroRNAs expression profiles in cardiovascular diseases. Biomed Res Int. 2014; 2014:985408.

7. Klimczak D, Paczek L, Jazdzewski K, Kuch M. MicroRNAs: Powerful regulators and potential diagnostic tools in cardiovascular disease. Kardiol Pol. 2015;73:1-6.

8. Anglicheau D, Muthukumar T, Suthanthiran M. MicroRNAs: Small RNAs with big effects. Transplantation. 2010;90:105-112.

9. Creemers EE, Tijsen AJ, Pinto YM. Circulating microRNAs: Novel biomarkers and extracellular communicators in cardiovascular disease? Circ Res. 2012;110:483-495.

10. Zhu H, Fan GC. Extracellular/circulating microRNAs and their potential role in cardiovascular disease. Am J Cardiovasc Dis. 2011;1: 138-149.

11. Dangwal S, Thum T. MicroRNA therapeutics in cardiovascular disease models. Annu Rev Pharmacol Toxicol. 2014;54:185-203.

12. Philippen LE, Dirkx E, Wit JB, Burggraaf K, de Windt LJ, da Costa Martins PA. Antisense MicroRNA Therapeutics in Cardiovascular Disease: Quo Vadis? Mol Ther. 2015;23(12):1810-1818.

13. Small EM, Olson EN. Pervasive roles of microRNAs in cardiovascular biology. Nature. 2011;469:336-342.

14. Porrello ER. MicroRNAs in cardiac development and regeneration. Clin Sci (Lond). 2013;125:151-166.

15. Zhao Y, Ransom JF, Li A, et al. Dysregulation of cardiogenesis, cardiac conduction, and cell cycle in mice lacking miRNA-1-2. Cell. 2007;129:303-317.

16. Holler KL, Hendershot TJ, Troy SE, Vincentz JW, Firulli AB, Howard MJ. Targeted deletion of Hand 2 in cardiac neural crest-derived cells influences cardiac gene expression and outflow tract development. Dev Biol. 2010;341:291-304.

17. Liu N, Bezprozvannaya S, Williams AH, et al. MicroRNA-133a regulates cardiomyocyte proliferation and suppresses smooth muscle gene expression in the heart. Genes Dev. 2008;22:3242-3254.

18. Boettger T, Braun T. A new level of complexity: The role of microRNAs in cardiovascular development. Circ Res. 2012;110:1000-1013.

19. van Rooij E, Sutherland LB, Qi X, Richardson JA, Hill J, Olson EN. Control of stress-dependent cardiac growth and gene expression by a microRNA. Science. 2007;316:575-579.

20. Porrello ER, Johnson BA, Aurora AB, et al. MiR-15 family regulates postnatal mitotic arrest of cardiomyocytes. Circ Res. 2011;109: 670-679.

21. Nishi H, Ono K, Iwanaga $Y$, et al. MicroRNA-15b modulates cellular ATP levels and degenerates mitochondria via Arl2 in neonatal rat cardiac myocytes. J Biol Chem. 2010;285:4920-4930.
22. Fuller AM, Qian L. MiRiad Roles for MicroRNAs in cardiac development and regeneration. Cells. 2014;3:724-750.

23. Porrello ER, Mahmoud Al, Simpson E, et al. Regulation of neonatal and adult mammalian heart regeneration by the miR-15 family. Proc Natl Acad Sci USA. 2013;110:187-192.

24. Yin VP, Lepilina A, Smith A, Poss KD. Regulation of zebrafish heart regeneration by miR-133. Dev Biol. 2012;365:319-327.

25. Eulalio $A$, Mano $M$, Dal Ferro $M$, et al. Functional screening identifies miRNAs inducing cardiac regeneration. Nature. 2012;492: 376-381.

26. Laflamme MA, Zbinden S, Epstein SE, Murry CE. Cell-based therapy for myocardial ischemia and infarction: Pathophysiological mechanisms. Annu Rev Pathol. 2007;2:307-339.

27. Glass C, Singla DK. MicroRNA-1 transfected embryonic stem cells enhance cardiac myocyte differentiation and inhibit apoptosis by modulating the PTEN/Akt pathway in the infarcted heart. Am J Physiol Heart Circ Physiol. 2011;301:2038-2049.

28. Hosoda T, Zheng H, Cabral-da-Silva M, et al. Human cardiac stem cell differentiation is regulated by a mircrine mechanism. Circulation. 2011;123:1287-1296.

29. Qian L, Huang $\mathrm{Y}$, Spencer $\mathrm{Cl}$, et al. In vivo reprogramming of murine cardiac fibroblasts into induced cardiomyocytes. Nature. 2012;485:593-598.

30. Jayawardena TM, Egemnazarov B, Finch EA, et al. MicroRNA-mediated in vitro and in vivo direct reprogramming of cardiac fibroblasts to cardiomyocytes. Circ Res. 2012;110:1465-1473.

31. Jayawardena TM, Finch EA, Zhang L, et al. MicroRNA induced cardiac reprogramming in vivo: Evidence for mature cardiac myocytes and improved cardiac function. Circ Res. 2015;116:418-424.

32. van Rooij E, Sutherland LB, Thatcher JE, et al. Dysregulation of microRNAs after myocardial infarction reveals a role of miR-29 in cardiac fibrosis. Proc Natl Acad Sci USA. 2008;105:13027-13032.

33. Rane S, He M, Sayed D, et al. Downregulation of miR-199a derepresses hypoxia-inducible factor-1alpha and Sirtuin 1 and recapitulates hypoxia preconditioning in cardiac myocytes. Circ Res. 2009; 104:879-886.

34. Mendell JT, Olson EN. MicroRNAs in stress signaling and human disease. Cell. 2012;148:1172-1187.

35. Cai WF, Liu GS, Lam CK, et al. Up-regulation of micro-RNA765 in human failing hearts is associated with post-transcriptional regulation of protein phosphatase inhibitor-1 and depressed contractility. Eur J Heart Fail. 2015;17:782-793.

36. Wahlquist $C$, Jeong $D$, Rojas-Munoz $A$, et al. Inhibition of miR25 improves cardiac contractility in the failing heart. Nature. 2014:508:531-535.

37. Xu M, Wu HD, Li RC, et al. Mir-24 regulates junctophilin-2 expression in cardiomyocytes. Circ Res. 2012;111:837-841.

38. Melman YF, Shah R, Danielson K, et al. Circulating MicroRNA-30d is associated with response to cardiac resynchronization therapy in heart failure and regulates cardiomyocyte apoptosis: A translational pilot study. Circulation. 2015;131:2202-2216.

39. Potus F, Ruffenach G, Dahou A, et al. Downregulation of MicroRNA-126 contributes to the failing right ventricle in pulmonary arterial hypertension. Circulation. 2015;132:932-943.

40. Halkein J, Tabruyn SP, Ricke-Hoch M, et al. MicroRNA-146a is a therapeutic target and biomarker for peripartum cardiomyopathy. J Clin Invest. 2013;123:2143-2154.

41. Cai B, Pan Z, Lu Y. The roles of microRNAs in heart diseases: A novel important regulator. Curr Med Chem. 2010;17:407-411.

42. Li YD, Hong YF, Yusufuaji Y, et al. Altered expression of hyperpolarization-activated cyclic nucleotide-gated channels and microRNA-1 and -133 in patients with age-associated atrial fibrilation. Mol Med Rep. 2015;12(3):3243-3248.

43. Shi L, Liao J, Liu B, Zeng F, Zhang L. Mechanisms and therapeutic potential of microRNAs in hypertension. Drug Discov Today. 2015;20:1188-1204.

44. Batkai S, Thum T. MicroRNAs in hypertension: Mechanisms and therapeutic targets. Curr Hypertens Rep. 2012;14:79-87.

45. Liao YC, Wang YS, Guo YC, Lin WL, Chang MH, Juo SH. Let-7g improves multiple endothelial functions through targeting transforming growth factor-beta and SIRT-1 signaling. J Am Coll Cardiol. 2014;63:1685-1694. 
46. Zhao N, Koenig SN, Trask AJ, et al. MicroRNA miR145 regulates TGFBR2 expression and matrix synthesis in vascular smooth muscle cells. Circ Res. 2015;116:23-34.

47. Climent M, Quintavalle M, Miragoli M, Chen J, Condorelli G, Elia L. TGFbeta triggers miR-143/145 transfer from smooth muscle cells to endothelial cells, thereby modulating vessel stabilization. Circ Res. 2015;116:1753-1764.

48. Rayner KJ, Moore KJ. MicroRNA control of high-density lipoprotein metabolism and function. Circ Res. 2014;114:183-192.

49. Janssen HL, Reesink HW, Lawitz EJ, et al. Treatment of HCV infection by targeting microRNA. N Engl J Med. 2013;368:1685-1694.

50. Farooqi AA, Fayyaz S, Shatynska-Mytsyk I, et al. Is miR-34a a wellequipped swordsman to conquer temple of molecular oncology? Chem Biol Drug Des. 2016;87(3):321-334. 